\title{
DEMOCRACIA, PARTICIPACIÓN Y FEMINISMO
}

\author{
Alejandra Castillo Vega
}




\section{ALEJANDRA CASTILLO VEGA}

Doctora en Filosofía. Profesora titular del Departamento de Filosofía de la Universidad Metropolitana de Ciencias de la Educación, UMCE. Directora de la Revista de Cultura "Papel Máquina". Es autora de "Feminismos en disputa" (2016); "Imagen, cuerpo" (2015); "Ars disyecta. Figuras para una corpo-política" (2014); "El desorden de la democracia. Partidos políticos de mujeres en Chile" (2014); "Nudos feministas. Política, filosofía, democracia" (2011); "Democracia, políticas de la presencia y paridad" (2011); "Julieta Kirkwood. Políticas del nombre propio" (2007); "La república masculina y la promesa igualitaria" (2005). Editora de Martina Barros, "Prólogo a la Esclavitud de la Mujer" (2009); y co-editora de "Arte, archivo y tecnología" (2012); "Re-escrituras de José Martí" (2008) y "Nación, Estado y cultura en América Latina" (2003). 


\title{
DEMOCRACIA, PARTICIPACIÓN Y FEMINISMO
}

\author{
"No hay democracia sin feminismo'. Descartando \\ las prioridades o contradicciones primarias o \\ secundarias, afirma la naturaleza constitutiva de toda \\ opresión que implica dominación, discriminación y \\ subordinación de las mujeres en el mundo privado y \\ público". \\ (Julieta Kirkwood, Serpolitica en Chile)
}

Lo que fue habitual hasta hace un tiempo en las discusiones sobre la democracia en Chile, ya no lo es más. Se suele decir que la búsqueda del consenso como bien principal del régimen democrático chileno habría terminado por vaciar a la democracia de todo contenido, dejando en su lugar sólo un conjunto de procedimientos eleccionarios enfrentados a la apatía y la baja participación. Sin embargo, nos equivocamos si pensamos que ésta, nuestra democracia, es meramente procedimental. El equívoco es pensar a la democracia en abstracción del marco sustantivo que la constituye y la dota de contenidos: la Constitución de 1980. Lo que se ha evidenciado con el paso de los gobiernos de la Concertación, y éste de la Nueva Mayoría, es el despliegue y puesta en práctica de su orden sustantivo ligado al ideario políticoeconómico neoliberal. Es por esa ligazón que nuestra democracia no es en absoluto "meramente" procedimental; cada uno de los conceptos que la describen reproduce una sociedad de mercado. ¿Qué decimos con derechos en este contexto? Un conjunto de bienes alcanzables dependiendo de la capacidad adquisitiva de cada quien, ahí está la educación y la salud para demostrarlo. ¿Qué decimos con participación? No otra cosa que acudir de tanto en tanto a las urnas para legitimar un proceso eleccionario. Salir de este equívoco nos obliga a darnos a la tarea de proponer otros modos de pensar los contenidos que describen a la democracia; esto implica, sin duda, proponer otras definiciones para una nueva Constitución. No es otra la intención de este ensayo que la de poner en discusión otros sentidos para la definición de una democracia que aún está por venir. Me concentraré, especialmente, en el vínculo entre democracia, participación y feminismo.

La democracia se dice en la participación. Es el orden en el que el número, la cifra, determina quién o quiénes representarán la voluntad del pueblo. Nada dice la participación de la diferencia de los sexos. El filósofo francés Jacques Rancière 
advierte, sin embargo, que la democracia es el nombre de una interrupción singular a la organización y distribución de los cuerpos en comunidad (Rancière, p. 139). La democracia no sería otra cosa que la interrupción de su funcionamiento por un singular dispositivo de subjetivación (Rancière, p. 140). Este dispositivo, si bien no es un aparato, sí es posible de describir como un conjunto de piezas cuyo funcionamiento, paradójicamente, más que iniciar o continuar el movimiento, lo detiene o, como señala Rancière, lo interrumpe. Las piezas de este engranaje son tres: el reconocimiento de la existencia de un orden de lo "en común"; el descalce entre ese orden y las partes (cuerpos) que lo componen; y el establecimiento de un litigio por el propio sentido del nombre de la política, esto es, de la democracia (Rancière, p. 141).

Si es así, y la democracia es siempre un régimen cuyo fundamento es la interrupción, qué ocurriría si esa interrupción tomara la particular forma de un cuestionamiento feminista: ¿tiene sexo la democracia? Esta pregunta no buscó responderla Rancière. No obstante, durante los mismos años en que Rancière escribía el seminal libro La Meséntente, destacadas teóricas y filósofas se dieron a la tarea de responderla. Ejemplares en este gesto son los cuestionamientos realizados por Iris Marion Young a la abstracción del orden democrático liberal, proponiendo una política de la diferencia de los cuerpos sexuados. Desde una perspectiva feminista materialista, Anna G. Jónasdóttir, a pesar de no estar de moda, repondrá el concepto de patriarcado moderno para volver visible la opresión que sufren las mujeres en los regímenes democráticos. En una línea teórica afín, Catharine Mackinnon propondrá, por su parte, los primeros lineamientos hacia una teoría feminista del Estado. ¿'Tiene sexo la democracia? nos podríamos preguntar nuevamente. Para estas autoras sin duda que la democracia tiene sexo y organiza lo común de la comunidad desde una ordenación androcéntrica. ¿Cómo interrumpir esta ordenación? Anne Phillips propondrá una política de la presencia.

No sin novedad para los estudios sobre la representación política, Anne Phillips elabora un modo de ver la participación volviendo cercanos dos modelos que hasta ese entonces se tenían por antagónicos: la democracia participativa y la democracia representativa. La antigua disputa figurada en la disyunción entre quiénes deciden o qué se decide —o más claramente dicho, cuál de aquellas posiciones es más relevante a la hora de describir un orden democrático - será desplazada por el concepto de "política de la presencia", que si bien se concibe como una reforma de la representación política, su punto de partida, sin embargo, se encuentra en una certera crítica respecto de la composición de los regímenes democráticos representativos. Esta composición delataba un preocupante déficit de participación de las mujeres en todas las instancias democráticas. De ahí la importancia de poner atención en quiénes participan. 
Fueron tres los argumentos con los que se ha defendido la necesidad de impulsar en términos políticos y teóricos esta política de la presencia. El primero de ellos apunta al orden de la representación simbólica. Si uno de los sexos tiene, marcadamente, más visibilidad que el otro, establece, implícitamente, un tutelaje sobre las decisiones del otro como si éste estuviera en minoría de edad. Es por esta lógica que las decisiones, posiciones y elecciones de las mujeres pasan "simbólicamente" por la aprobación de sus padres, hermanos o hijos, pese a haber obtenido sus derechos políticos ya hace bastante tiempo. Siguiendo la hebra que este argumento deja, Phillips indica que "por mucho que el espíritu público anime a sus mentores, esta infantilización de grandes sectores de la ciudadanía es difícilmente compatible con la democracia" (Phillips, p. 241).

El segundo argumento busca hacer de la política un lugar para la defensa de los intereses de los grupos excluidos o marginados. La política de contenidos ha terminado por demostrar sus limitaciones a la hora de defender los intereses de los grupos excluidos del orden político. En el caso de identidades constituidas en el cruce de las variantes de raza-género, esta exclusión implica, también, estar excluido en términos económicos. El paso desde una política de contenidos a una de la presencia implica un cambio en la representación en el sentido que los representantes de los grupos excluidos hacen de los intereses de sus representados los suyos propios en la medida que su participación y sus intereses son coincidentes. Así descrita, la política de la presencia evidencia con mayor fuerza el "mandato vinculante" que es supuesto entre el electorado y su representante. Este argumento vuelve muy relevante quiénes sean nuestros representantes. Volviendo tangentes las políticas de la representación y las participativas, Anne Phillips indica que "los miembros de los grupos desfavorecidos necesitan tener defensores con más empuje y dinamismo en la escena pública. No se trata de que la gente sólo actúe para sí misma" (Phillips, p. 245). Es importante señalar que este argumento apunta, por un lado, a redefinir las democracias representativas no como "meramente" procedimentales, sino que como democracias participativas ligadas a intereses comunes y locales; y, por otro, este argumento participativo puede ser visto como un freno a la creciente corrupción de los regímenes de orden representacional. Cabe destacar que esta alteración del orden procedimental desde sus propios mecanismos y procedimientos fue lo que alentó a bautizar a esta reforma como "reforma revolucionaria".

Por último, la política de la presencia se anuncia como la transformación de un orden político y social injusto. Para Anne Phillips es éste, y no otro, el argumento más importante a favor de la política de la presencia. Para esta autora es simplemente inaceptable e injusto que los hombres monopolicen la representación política de las mujeres. De no haber serios obstáculos para la participación de diversos grupos de ciudadanos y ciudadanas, la composición de las asambleas elegidas no 
evidenciaría un patrón de participación que beneficiara notoriamente a un grupo sobre otros (Phillips, p. 245). La razón que subyace para la sub-representación de las mujeres en política es la mantención del inconfeso argumento de la división sexual del trabajo entendido como una ordenación natural. Volviendo explícita la motivación feminista de su posicionamiento, Phillips afirma que "la diferencia sexual no es un mero 'hecho' de la biología humana, sino que gira aún en torno de una diferenciación entre hombres y mujeres relativamente incontrovertida" (Phillips, p. 245). La naturalización de la diferencia sexual como descriptora de dos formas de vida, coincidente una con la esfera de lo privado/doméstico y la otra con la esfera de lo público/político, excluye a las mujeres de antemano del espacio de la participación. De ahí la necesidad de poner atención no sólo a los mecanismos para la participación, sino que también en quiénes nos representan. Variando la pregunta con la que comenzábamos este ensayo: ¿Le importa el sexo a la democracia? Sí, y mucho, parece responder Anne Phillips, por ello cree necesario transformar la representación con políticas de la presencia cuyas formas han sido, principalmente, la paridad y la ley de cuotas.

Si bien esta reforma revolucionaria, como se la llamó en sus inicios, complejiza el problema de la representación llevándolo más allá del debate sobre las "preferencias", no parece poner atención al grupo de mujeres que se vería beneficiado por esta política. Dicho de otro modo, más allá de la igualdad de género que esta política promueve, parece ser irrelevante para su representación saber cuál es la clase social a la que pertenecerían estas mujeres: al momento de representar "política de mujeres", ¿todas las mujeres tendrán los mismos intereses? ¿Todas las mujeres tendrían la misma posibilidad de participar? Es frente a este tipo de preguntas que esta política de la presencia evidenció prontamente dos problemas. Uno de ellos tenía relación con el necesario componente esencialista en la definición de este mecanismo procedimental. Esta política de la presencia favorecía la participación de mujeres en política para corregir la ordenación androcéntrica dominante. Sin embargo, presupone necesariamente una política de la identidad fuerte, esto es, implica presuponer que las mujeres comparten las mismas motivaciones e intereses. Sin poner atención en los componentes de clase o étnicos, la política de la presencia debe asumir una definición de "mujeres" naturalizada haciendo de su cuerpo el principal soporte y promotor de sus políticas. No es extraño por ello que "naturalmente" las mujeres de distintos colores políticos en asambleas electas tomen las banderas de la infancia, la maternidad, el apego y el cuidado como principales temas de legislación.

Un segundo problema que dejó ver esta particular política de mujeres tuvo relación con la clase social. Las políticas de la presencia, al ser cupos dejados para la participación exclusiva de mujeres, ya sea en partidos políticos o para optar a 
escaños en el Congreso, facilitan la participación de mujeres que, de algún modo, ya tienen presencia en otros ámbitos. Las mujeres que logran visibilidad por este mecanismo ya son visibles en el espacio de lo social.

Este problema es aún más notorio cuando estas políticas de la presencia son asumidas desde "arriba", esto es, desde instancias gubernamentales no eleccionarias. Tomemos por caso nuestro país. Será durante el Gobierno de Ricardo Lagos (2000-2005) que se intentará revitalizar y darle un nuevo rostro a la política de la Concertación, incorporando cinco ministras en su gobierno, hecho inédito en la política chilena. En este sentido, Lagos comenta que:

En el primer gabinete del Presidente Aylwin se nombró a una mujer a cargo de los temas de la mujer, por supuesto, y hubo un poco más de participación femenina entre ellos. A mí me pareció que había que dar un mensaje potente de incorporación de la mujer: en mi primer gabinete, de 16 hubo cinco ministras. Hicimos algo parecido a nivel de intendentes. Creo que esto fue un elemento importante. Pero cuando dijimos pongamos cinco mujeres, no fueron cinco para algo determinado, sino porque quería dar un salto que fuera notorio (Fernández, p. 48).

Esta política de la presencia luego será retomada con mayor vigor durante el primer Gobierno de Michelle Bachelet (2006-2010). Debo destacar que esta demanda por más presencia de mujeres en dicho periodo quedará definida en las palabras de paridad y excelencia. Se presenta con cierto énfasis la "idea de excelencia" para dejar en claro que lo que se pide no es, simplemente, "más mujeres"; no es la ley del número a lo que apelan, sino que a más mujeres tanto o más capacitadas que cualquier hombre en el espacio de la política. Es por ello que junto a la experiencia política de las ministras del gabinete paritario se presentarán también sus biografías académicas y profesionales. Diplomas, maestrías, doctorados y conocimientos de idiomas extranjeros serán pruebas necesarias de competencias y habilidades para desarrollar el cargo político asignado (Castillo, p. 13-43). De esta manera, la política de la presencia quedará reducida a una azarosa política presidencial que privilegiará a mujeres pertenecientes a alguna élite (económica, familiar, política, etcétera) ¿Qué ocurre con el resto de las mujeres? ¿Cómo corregir la falta de participación de las mujeres no pertenecientes a la élite? Es frente a estos dos problemas que pareciera quedar en falta la política de la presencia.

La filósofa norteamericana Nancy Fraser ha notado que este déficit de participación no sólo es relevante para la transformación de la representación política, sino que este déficit implica por sobre todo injusticia social. Así lo ha señalado en Fortunes of Feminism. From State-Managed Capitalism to Neoliberal Crisis. 
En este libro propone un interesante giro al concepto de paridad. Debe ser dicho que la "paridad", en el contexto que este libro provee, no queda restringida al aseguramiento de la presencia de mujeres en las diversas instancias eleccionarias, sino que se concibe como un concepto ligado a la justicia de género entendida ésta como "paridad participativa". Explicando este concepto, Nancy Fraser indica:

En Francia, parité se aplica sólo a un campo de interacción: las campañas electorales para obtener escaños en las asambleas legislativas. Para mí, sin embargo, la paridad es aplicable a toda la vida social. La justicia requiere, por lo tanto, paridad de participación en una multiplicidad de campos de interacción, incluidos los mercados de trabajo, las relaciones sexuales, la vida de familia, las esferas públicas y las asociaciones voluntarias en la sociedad civil. En cada campo, sin embargo, la participación significa algo distinto (Fraser, p. 197).

Desde esta perspectiva, la justicia se entenderá como soluciones sociales que hagan posible que todos los miembros (adultos) de una comunidad dada puedan interactuar entre sí como iguales. Para que este particular gesto paritario ocurra son necesarias dos condiciones. Una de orden distributivo y la otra de orden del reconocimiento. La justicia como paridad participativa exige la implementación de ambos órdenes en términos relacionales. Ninguno de ellos es suficiente por sí mismo para hacer de la democracia un régimen participativo.

Una primera condición es de índole económica. Volviendo a las exigencias del feminismo socialista, en que la igualdad se trama con redistribución, Nancy Fraser afirmará que "la distribución de los recursos materiales debe ser tal que garantice la independencia y la 'voz' de los participantes” (Fraser, p. 195). Esta condición no es, en ningún caso, un "bono", ya sea de locomoción o de alimentación, para "participar" en los actos eleccionarios, sino que, muy por el contrario, es una condición que busca transformar la injusticia social que promueve la participación de algunos y algunas e impide la participación de otros y otras. Esta condición económica busca poner al margen del juego de la política las desigualdades que hacen imposible la participación.

En este punto, Nancy Fraser define la paridad como participación en tanto soluciones sociales que excluyan la institucionalización de la "privación, la explotación y excesivas disparidades de riqueza, renta y tiempo de ocio, negando así a algunas personas los medios y las oportunidades para interactuar con otras como iguales" (Fraser, p. 195). Esta condición primera vuelve evidente que la participación implica igualdad. De tal modo que un "gobierno paritario" — por tomar aquel slogan con el que se da inicio al primer Gobierno de Bachelet- no significa "más mujeres capacitadas", sino que transformar los impedimentos económicos y sociales que 
hacen difícil, si no imposible, la participación de todas las mujeres en los distintos ámbitos de lo político y social. Esta redefinición del concepto de paridad pone de nuevo en el tapete debates que se creyeron resueltos por los feminismos de la acción afirmativa. Debates como la persistencia, todavía hoy, de una soterrada división sexual del trabajo que no sólo diferencia áreas y dominios laborales, sino que experiencias y formas de vida diferenciadas y excluyentes (Federici, p. 196). O como aquel otro relativo al trabajo del cuidado (crianza, trabajo doméstico, cuidado de la tercera edad), que es mayoritariamente realizado de forma impaga por mujeres (Morini, p. 39). A estos debates clásicos del feminismo radical se suman hoy la precarización laboral y la creciente feminización de la pobreza (Pérez, p.36).

La segunda condición que propone Fraser para la puesta en práctica de una justicia como paridad participativa se sitúa en el plano de lo cultural-social. Esta condición indica que las pautas de comportamiento de los miembros de una comunidad dada deben ser respetuosas de la participación de unas y otras (Fraser, p. 195). La premisa de esta condición es la exclusión de los patrones institucionalizados que "nieguen a algunas personas la categoría de plenos participantes en la interacción, ya sea cargándolos de una excesiva 'diferencia' o no reconociendo su singularidad" (Fraser, p. 195). Esta segunda condición va en dirección de las políticas del reconocimiento, esto es, políticas que transformen status y jerarquías cuando éstas implican denigración y daño a quienes son reconocidos por tales status y jerarquías (Fraser; Honneth, p. 21). En esta línea estarían, por ejemplo, todas aquellas políticas tendientes a erradicar el sexismo, el machismo y la violencia simbólica. Transformar estos status y jerarquías es obligatorio para hablar de participación en democracia. Para algunas teóricas y políticas, la transformación de la esfera de lo político y social desde un signo feminista implica volver explícito que ese status — desde el cual las mujeres son reconocidas tanto en el espacio privado como en el público- es definido por una ordenación de género jerárquica, cuya escala se describe desde una supremacía de lo masculino. Esta es la perspectiva del feminismo jurídico de Catharine Mackinnon. Esta desigualdad intrínseca, promovida por el propio binarismo de género, reproduciría un orden de violencia cotidiana hacia las mujeres. Extremando el vínculo entre género, sexualidad y violencia, Mackinnon llega a afirmar que:

Todas las maneras en que las mujeres suprimidas y dominadas - restringidas, invalidadas, violadas, objetivadas - se reconocen como lo que el sexo es para las mujeres y como el significado y el contenido de la feminidad. Si esto es así, la sexualidad en sí misma ya no queda desvinculada del estatus de segunda clase de las mujeres. La violencia sexual ya no puede ser categorizada como violencia y no sexo (Mackinnon, p. 20-21). 
De ahí que la transformación de tales status y jerarquías de las que habla Nancy Fraser implique poner atención a los marcos institucionales que favorecen la reproducción de un orden sexista y violento hacia las mujeres y promover, a su vez, cambios de signo feminista en las instituciones mismas — pensemos en las universidades, por ejemplo- en lo que tiene que ver con sus disposiciones jurídicas. Sin embargo, esto no es todo. Es necesario transformar también los discursos que han definido y limitado los cuerpos bajo una ordenación de género heteronormativa, puesto que de ahí deriva la ordenación jerárquica que describe a algunos cuerpos en desnivel, en una caída. Es desde esta perspectiva que debiéramos preguntarnos, aun cuando esta pregunta, necesariamente, nos lleve hacia otros feminismos: "¿Qué cuerpos llegan a importar? ¿Y por qué?” (Butler, p. 14).

Si lo que nos interesa es aportar en la construcción de otra democracia para Chile no debiésemos olvidar aquello que señalara Julieta Kirkwood en los años '80: no hay democracia sin feminismo (Kirkwood, p. 222). A pesar de la parquedad de la fórmula, no yerra en hacer visibles cuerpos y formas de opresión. Tomar en serio aquella fórmula implica una interrupción del orden de lo común que da sustento a nuestro orden democrático. Sólo a partir de este cuestionamiento podremos pensar seriamente el vínculo entre democracia y participación. 


\section{REFERENCIAS}

Butler, Judith, Cuerpos que importan. Sobre los límites materiales y discursivos del "sexo", Buenos Aires, Paidós, 2002.

Castillo, Alejandra, Democracia, política de la presencia y paridad. Estudio sobre participación política de mujeres en el Ejecutivo (2006-2010), Santiago, Corporación Humanas Ediciones, 2011.

Federeci, Silvia, Caliban y la bruja. Mujeres, cuerpo y acumulación originaria, Buenos Aires, Editorial Tinta limón, 2011.

Fernández, María de los Ángeles, "Mujer y política: entrevista al Presidente Lagos" en Bienvenida, Paridad, Santiago, Cuarto Propio.

Fraser, Nancy, Fortunes of feminism. From State-Managed Capitalism to Neoliberal Crisis, Londres, Verso, 2013.

Fraser, Nancy, Fortunas del feminismo. Del capitalismo gestionado por el Estado a la crisis neoliberal, Quito-Madrid, Instituto de Altos Estudios nacionales del EcuadorTraficantes de sueños, 2015.

Fraser, Nancy y Axel Honneth, ¿Redistribución o Reconocimiento? Un debate politicofilosófico, Madrid, Editorial Ediciones Morata, 2006.

Jonnasdottir, Anna, Love, Power and Political Interests. Towards a Theory of Patriarchy in Contemporary Western Societies, Philadelphia, Temple University Press, 1993.

Kirkwood, Julieta, Ser política en Chile. Los nudos de la sabiduría feminista, Santiago, Cuarto propio, 1986.

Mackinnon, Catharine, Feminismo inmodificado. Discursos sobre la vida y el derecho, Buenos Aires, Siglo XXI Editores, 2014

Morini, Cristina, Por amor o la fuerza. Feminización del trabajo y biopolítica del cuerpo, Madrid, Traficantes de sueños, 2014.

Phillips, Anne, Politics of Presence, Oxford, Clarendon Press, 1995.

Phillips, Anne, "La política de la presencia: la reforma de la representación política", Soledad García y Steven Lukes (comps.), Ciudadania: justicia social, identidad y participación, Madrid, Siglo XXI Editores, 1999.

Phillips, Anne, "Democracy and Representation: Or, Why Should matter Who our Representatives Are? Anne Phillips (ed.), Feminism and Politics, Oxford, Oxford University Press, 2004.

Rancière, Jacques, La Meséntente. Politique et Philosophie, París, Galilée, 1995.

Young, Iris M., Justice and the Politics of Difference, Princeton, Princeton University Press, 1990. 